\title{
The short and long-term impact of educational intervention on pharmacovigilance awareness in second year MBBS students: a questionnaire-based survey
}

\author{
Lavanya S. Honnagangadevaru*, Kalpana Lakshmanegowda
}

Department of Pharmacology, BGS Global Institute of Medical Sciences, Bangalore, Karnataka, India

Received: 15 January 2020

Accepted: 02 March 2020

\section{*Correspondence:}

Dr. Lavanya S. Honnagangadevaru,

Email: lavanyasumanthraj@gmail.com

Copyright: ( $)$ the author(s), publisher and licensee Medip Academy. This is an open-access article distributed under the terms of the Creative Commons Attribution Non-Commercial License, which permits unrestricted non-commercial use, distribution, and reproduction in any medium, provided the original work is properly cited.

\begin{abstract}
Background: Under-reporting of adverse drug reactions (ADRs) is a burning issue globally. Sensitizing medical students to the concepts of pharmacovigilance in their early years through regular training holds the key for successfully perpetuating ADR reporting culture. This study attempted to assess the short and long-term impact of interventional training programs on the knowledge and perception of Pharmacovigilance amongst II MBBS students. Methods: It was a Cross-sectional, interventional, questionnaire-based study. Second MBBS, new entrants $(n=127)$ were tested for their awareness regarding pharmacovigilance knowledge using pre-validated questionnaires at entry point and following regular training sessions, at 1 week (short-term) and at 1 year (long-term). Students' perceptions towards ADR monitoring were also recorded. Descriptive statistics, Chi-square test, Independent t-test/MannWhitney-U test for identifying mean difference and paired-t-test/Wilcoxon signed rank test for paired data was used for quantitative and qualitative data analysis accordingly.

Results: Significant improvement in scores following training was seen at both short $(10.8 \pm 1.914)$ and long term (11.63 \pm 1.516$)$ compared to base-line scores (6.07 \pm 2.036$)$. There was significant increase in long-term scores compared to short-term scores $(<0.001)$. Female students performed better than their male counterparts. Majority of students positively perceived the need for ADR monitoring and reporting.

Conclusions: Pharmacovigilance training increases students' knowledge significantly. Strengthening the existing pharmacovigilance activities through regular refresher training programs and adequate sequencing in curriculum not only maintains but improves students' knowledge significantly.
\end{abstract}

Keywords: Awareness, Educational intervention, Impact, Pharmacovigilance, Short and long-term

\section{INTRODUCTION}

It is a well known fact that 'No drug is inherently safe'; unless it has no effect at all. ${ }^{1}$ Though considered a boon for humanity, drugs can impart adverse effects. In this regard, drug safety monitoring becomes an indispensable facet in ensuring effective use of medicines and providing quality healthcare. Pharmacovigilance, the science which primarily deals with adverse drug reactions (ADR) monitoring is an integral part of patient care. ${ }^{1}$ Good pharmacovigilance practice allows for the effective communication of medication associated risks, intelligent and evidence-based use of medicines and has the potential for preventing many adverse reactions. ${ }^{2}$

Despite the progress that has been made in pharmacovigilance through various measures initiated by the regulatory bodies worldwide, underreporting of ADRs is the biggest problem experienced globally. ${ }^{3}$ Establishing the culture of ADR reporting is a challenge for all medical colleges. In this regard, sensitizing students and resident doctors is of utmost importance. 
Targeting the most important taskforce group of medical students and catching them young so that they are knowledgeable and motivated enough to report any ADRs encountered in their future practice, would be a shot in the arm for the efforts of the Pharmacovigilance Programme of India (PvPI), initiated by the Central Drugs Standard Control Organization, New Delhi in $2010 .{ }^{4}$ Although numerous studies have been conducted in the past to know the awareness levels amongst the medical fraternity, a very few have aimed to assess the outcome of interventional training programs on the knowledge and perception of pharmacovigilance in medical students. ${ }^{5,6}$ Hence this study was aimed to assess the short and long-term impact of training programs on the awareness and knowledge of pharmacovigilance amongst II MBBS students.

\section{METHODS}

\section{Study setting}

This cross-sectional, interventional, questionnaire-based study was conducted in the Department of Pharmacology, during the academic year 2018-2019. Students in second professional MBBS $(n=146)$ were invited to participate in the study. The study was conducted according to Declaration of Helsinki guidelines. Informed consent was obtained and the students were informed that attending the sessions on pharmacovigilance during theory and practical sessions would be mandatory as pharmacovigilance was a part of undergraduate pharmacology curriculum. However, the students were assured that their participation in the questionnaire-based study was purely voluntary and that confidentiality would be maintained. Ethical clearance was obtained from the Institutional Ethics Committee.

\section{Methodology}

At the time of entry in to II MBBS (during the $1^{\text {st }}$ week) a pre-validated questionnaire was administered to the study participants. This questionnaire consisted of 15-items to assess student's knowledge about pharmacovigilance. The response format included multiple choice questions in which the students were asked to choose the correct answer from provided list of options. Each right answer was awarded one mark and the maximum score was 15 . The scores obtained were considered as pre-test scores and tabulated. Following this an educational intervention was designed in the form of interactive lectures and hands-on training by trained faculty to facilitate the transfer of knowledge of pharmacovigilance and ADR reporting. The learning objectives mainly focused on pharmacovigilance and its objectives, essentials of ADR reporting, Vigiflow database, classification and incidence of ADRs, role of health-care professionals in reporting of suspected ADRs in addition to the economic and epidemiological relevance of ADR reporting and its effect on patient health care. After 7 days of training, the students were administered the 15 -item questionnaire to measure changes in their pharmacovigilance knowledge, if any. The scores obtained were tabulated as Post-Test 1 scores, which reflected the impact of short- term training.

As previously mentioned, pharmacovigilance is a part of undergraduate pharmacology curriculum and hence ADR case reports that were reported to the ADR monitoring Centre of the Institution were discussed during practical sessions throughout the year. In addition the students were encouraged to report any suspected ADRs during their clinical postings using the Central Drugs Standard Control Organisation (CDSCO) suspected ADR reporting form. At the end of 1 year of reinforcement of concepts, students were administered the 15-item questionnaire again and the scores obtained were tabulated as Post-Test 2 . These scores reflected the impact of long-term training. To obtain the students' perspectives about ADR monitoring and reporting, they were administered a 7item pre-validated questionnaire that had to be answered on a 5-point Likert scale with the categories "strongly agree"- (score 5) to "strongly disagree"- (score 1) at the end of the study period (Figure 1).

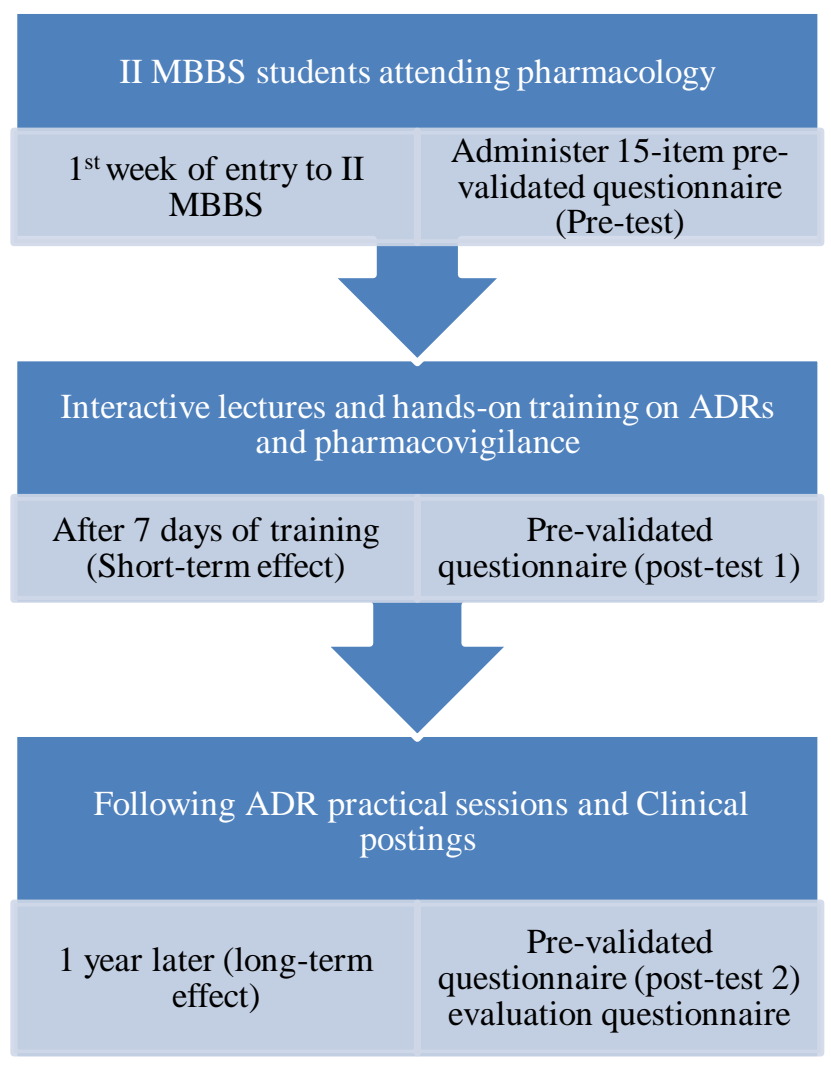

Figure 1: Study setting and methodology.

\section{Statistical analysis}

Data were entered into Microsoft excel data sheet and were analyzed using SPSS 22 version software. Categorical data were represented as frequencies and proportions. Chi-square test was used as test of significance for qualitative data. 
Continuous data was represented as mean and standard deviation. Independent $t$ test or Mann Whitney $U$ test was used as test of significance to identify the mean difference between two quantitative variables and qualitative variables respectively. Paired $t$ test or Wilcoxon Signed rank test is the test of significance for paired data such as pre-test and post-test for quantitative and qualitative data respectively. $\mathrm{P}<0.05$ was considered as statistically significant after assuming all the rules of statistical tests.

\section{RESULTS}

Out of 146 students attending pharmacology classes, a total of 127 students $(86.98 \%)$ completed both the pre and post-test questionnaires and perception questionnaires. Of these, majority were females accounting to $56.6 \%(n=72)$ and males constituted $43.4 \%(n=55)$. The findings are as follows.

\section{Pre-test and post-test score comparison}

In the study mean pre-test score was $6.07 \pm 2.036$, mean post-test score 1 was $10.83 \pm 1.914$ and mean post-test score 2 was $11.63 \pm 1.516$. There was significant increase in Post-test score 1 compared to pre-test score $(<0.001)$ and post-test score 2 compared to post-test score 1 $(<0.001)$ as shown in Figure 2.

In addition, an analysis was done to see if there was any gender difference with reference to the test scores. In the study there was no significant difference in mean pre-test score between males and females. However, there was significant difference in mean post-test score 1 and posttest score 2 between males and females. Females had higher post-test score 1 and score 2 compared to males as shown in Figure 3.

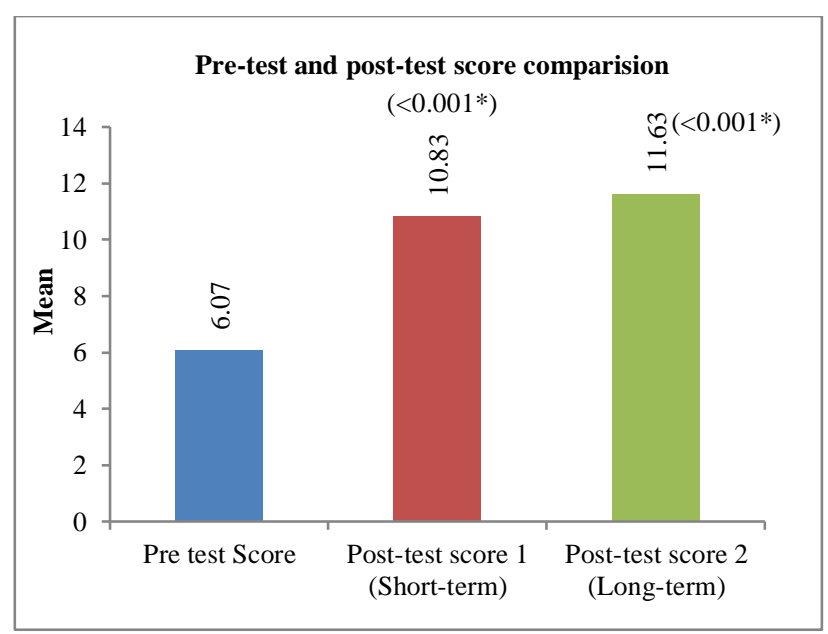

Figure 2: Pre-test and post-test score comparison.

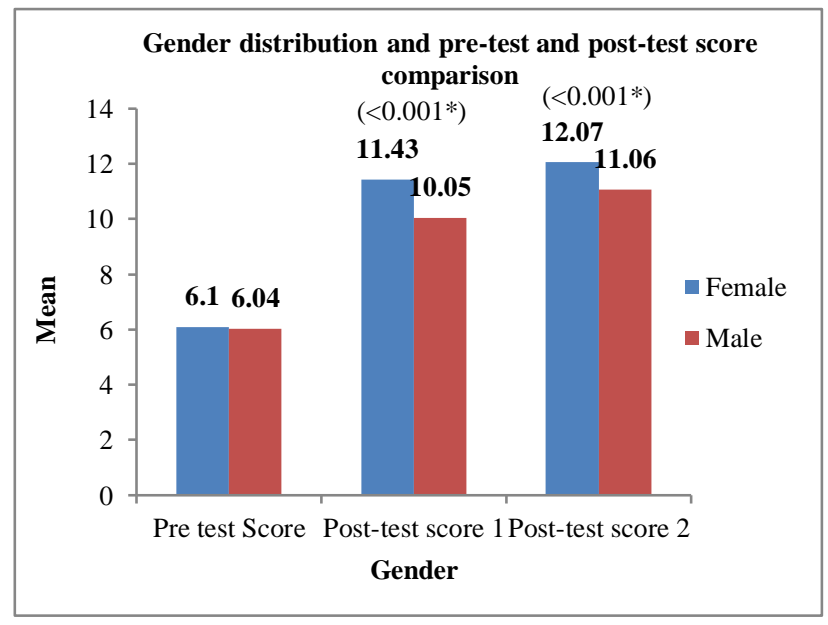

Figure 3: Pre-test and post-test score comparison with respect to gender distribution.

Table 1: Perception questionnaire distribution among study participants.

\begin{tabular}{|c|c|c|c|c|c|}
\hline Parameter & $\begin{array}{l}\text { Strongly } \\
\text { agree }\end{array}$ & Agree & Neutral & Disagree & $\begin{array}{l}\text { Strongly } \\
\text { disagree }\end{array}$ \\
\hline $\begin{array}{l}\text { P1: Reporting of ADRs is a part of professional } \\
\text { obligation of healthcare professionals }\end{array}$ & $53(42.1 \%)$ & $72(57.1 \%)$ & $1(0.8 \%)$ & 0 & 0 \\
\hline $\begin{array}{l}\text { P2: Pharmacovigilance and ADR monitoring should } \\
\text { be a part of undergraduate curriculum }\end{array}$ & $63(50 \%)$ & $51(40.5 \%)$ & $7(5.6 \%)$ & $5(4 \%)$ & 0 \\
\hline $\begin{array}{l}\text { P3: ADR reporting by medical fraternity makes a } \\
\text { significant contribution to the reporting system }\end{array}$ & $57(45.2 \%)$ & $66(52.4 \%)$ & $3(2.4 \%)$ & 0 & 0 \\
\hline $\begin{array}{l}\text { P4: ADR Surveillance system benefits both patients } \\
\text { and doctors }\end{array}$ & $55(43.7 \%)$ & $68(54 \%)$ & $3(2.4 \%)$ & 0 & 0 \\
\hline $\begin{array}{l}\text { P5: Medical students can play an important role in } \\
\text { pharmacovigilance system }\end{array}$ & $63(50 \%)$ & $53(42.1 \%)$ & $10(7.9 \%)$ & 0 & 0 \\
\hline P6: I am confident about the format of ADR reporting & $70(55.6 \%)$ & $47(37.3 \%)$ & $9(7.1 \%)$ & 0 & 0 \\
\hline $\begin{array}{l}\text { P7: I am motivated to report any ADRs noticeable in } \\
\text { my future practice }\end{array}$ & $66(52.4 \%)$ & $52(41.3 \%)$ & $7(5.6 \%)$ & $1(0.8 \%)$ & 0 \\
\hline
\end{tabular}




\section{Students' perceptions towards ADR monitoring and reporting}

In the study for parameters P2, P5, P6, P7, majority strongly agreed $(50 \%, 50 \%, 55.6 \%$ and $52.4 \%$ respectively) and for $\mathrm{P} 1, \mathrm{P} 3$ and $\mathrm{P} 4$ majority of them agreed $(57.1 \%, 52.4 \%$ and $54 \%$ respectively) as shown in Table 1.

There was no significant difference with respect to the perception questionnaire between males and females.

\section{DISCUSSION}

Pharmacovigilance activities have been the cornerstone of healthcare system for ensuring patient safety and must be an indigenous part of healthcare institutes. However ignorance, lack of training and motivation in reporting of ADRs are the major obstacles in achieving the objectives of National PV programmes. ${ }^{4}$ Lopez-Gonzalez et al, mentioned that "medical specialty was the most closely associated profession with under-reporting in $76 \%$ of studies". ${ }^{3}$ It is evident from various studies that reporting of ADRs by healthcare professionals increased after training. ${ }^{5,7}$ Hence, medical students' training holds the key to improved reporting rates.

We evaluated the knowledge of medicos regarding the various pharmacovigilance practices and the PvPI using pre and post-test questionnaires. The mean pre-test score was low irrespective of the gender, since the students were relatively naive to the concepts of Pharmacovigilance at the entry point to II year MBBS. However, following educational intervention, the posttest scores after 1 week of training (short-term effect) significantly improved when compared to the base-line scores. These findings are in concurrence with two other studies conducted by Arici et al and Ganesan et al. ${ }^{7,8}$

The post-test scores conducted at the end of 1 year (longterm effect) showed significantly high scores when compared to both pre-test and post-test score 1. This finding is in contrast to the study conducted by Arici et al, which demonstrated that improvement in post-test scores decreased significantly in the long-term (although higher than the base-line scores). ${ }^{8}$ Time and learning retention studies have suggested that "students do not retain the information acquired in a class for long after an exam" and students' understanding, or knowledge, may deteriorate over time in a non-linear manner. ${ }^{9}$ Fisher et al, in their study have confirmed a temporal degradation of clinical skills in medical students and have emphasized the need for refresher training for long-term retention. ${ }^{10}$ ADR reporting is a clinical skill that needs to be acquired by medical students through repeated teaching-learning practices. The same principle was applied during our study with adequate sequencing of pharmacovigilance classes during the II MBBS course in the form of ADR reporting exercises during pharmacology practical sessions and also by encouraging the students to report any ADRs encountered during their clinical postings. The reinforcement of concepts over time improves their comprehension and cognition, thus reduces the attrition rates as suggested by Semb et al. ${ }^{11}$ Present findings echo the observations made by Arici et al that "the effects of the educational intervention were temporary and hence regular PV retraining is essential". ${ }^{8}$

Further, our study also evaluated the gender difference with respect to pharmacovigilance awareness and training and found significantly higher scores following educational intervention in females than the male counterparts, despite identical and insignificant pre-test scores. These findings were in contrast to a study conducted by Othman et al where male students were more knowledgeable than females. ${ }^{12}$ However, the higher scores in females as seen in our study may be explained by considering the gender differences in neurobiological processes involved in memory functions as suggested by Loprinzi et al. ${ }^{13}$

The subjective segment of our study further evaluated the students' perception towards ADR monitoring and reporting. It was heartening to see that majority of students irrespective of gender owned up the responsibility of PV by agreeing to their role in ADR monitoring as a part of professional obligation for the betterment of both doctors and patients. Majority emphasized the need for curricular implementation and also confirmed high degree of confidence and motivation in future reporting. These findings are in alignment with numerous other studies conducted on students and healthcare professionals. ${ }^{14,15}$

\section{Strengths and limitations}

Most of the studies have focused on determining the awareness levels of pharmacovigilance among healthcare givers and the effect of educational intervention on a short term basis. However the present study analyses the impact of repeated pharmacovigilance training on both short and long-term basis; in addition to obtaining students' perceptions. Furthermore, this study was focused on II year MBBS students who are the future prescribers. Sensitizing them to the concepts of pharmacovigilance at an early phase and reiterating the concepts at regular intervals may have a positive impact on their comprehension, retention and subsequent reporting of ADRs in their future practice. However, the study was conducted in a guarded and supportive classroom setting. It would be interesting to see how much of this increased knowledge or behavioural change translates to actual ADR reporting in the real-life scenario.

\section{CONCLUSION}

Adverse drug reaction monitoring and reporting has remained the cornerstone of pharmacovigilance. However inadequate awareness about the pharmacovigilance system among the healthcare professionals is the leading 
cause of under reporting of ADRs. Undergraduate medical students are potential prospective caregivers who need to be sensitized at an early phase of their careers through adequate PV training to perpetuate reporting culture. The effects of educational intervention may be temporary. Hence existing pharmacovigilance activities must be strengthened by refresher training programs and adequate sequencing in curriculum.

\section{ACKNOWLEDGEMENTS}

The authors would like to thank the Management, Staff and Students, Department of Pharmacology, BGSGIMS for their encouragement and active participation in the study.

Funding: No funding sources

Conflict of interest: None declared

Ethical approval: The study was approved by the Institutional Ethics Committee

\section{REFERENCES}

1. Sattigeri BM, Moorthy V. Pharmacovigilance: Its clinical implications in health care management. J Integr Health Sci. 2014;2:33-5.

2. World Health Organization. Quality Assurance and Safety of Medicines Team. (2006). The safety of medicines in public health programmes: pharmacovigilance, an essential tool. World Health Organization. Available from: https://apps.who.int/ iris/handle/10665/43384. Accessed on 31 October 2019.

3. Lopez-Gonzalez E, Herdeiro MT, Figueiras A. Determinants of under-reporting of adverse drug reactions: a systematic review. Drug Safety. 2009;32(1):19-31.

4. Singh J, Singh H, Rohilla R, Kumar R, Gautam CS. Lack of awareness of pharmacovigilance among young health-care professionals in India: An issue requiring urgent intervention. Int $\mathbf{J}$ Appl Basic Med Res. 2018;8(3):158-63.

5. Gupta SK, Nayak RP, Shivaranjani R, Vidyarthi SK. A questionnaire study on the knowledge, attitude, and the practice of pharmacovigilance among the healthcare professionals in a teaching hospital in South India. Perspect Clin Res. 2015;6:45-52.

6. Rehan HS, Sah RK, Chopra D. Comparison of knowledge, attitude and practices of resident doctors and nurses on adverse drug reaction monitoring and reporting in a tertiary care hospital. Indian $\mathbf{J}$ Pharmacol. 2012;44:699-703.

7. Ganesan S, Sandhiya S, Reddy KC, Subrahmanyam DK, Adithan C. The impact of the educational intervention on knowledge, attitude, and practice of pharmacovigilance toward adverse drug reactions reporting among health-care professionals in a tertiary care hospital in South India. J Nat Sci Biol Med. 2017;8(2):203-9.

8. Arici MA, Gelal A, Demiral Y, Tuncok Y. Short and long-term impact of pharmacovigilance training on the pharmacovigilance knowledge of medical students. Indian J Pharmacol. 2015;47(4):436-9.

9. Kamuche, FU, Ledman RE. Relationship of time and learning retention. J Coll Teach Learn. 2005;2(8):258.

10. Fisher J, Viscusi R, Ratesic A, Johnstone C, Kelley $\mathrm{R}$, Tegethoff AM, et al. Clinical skills temporal degradation assessment in undergraduate medical education. J Adv Med Educ Prof. 2018;6(1):1-5.

11. Semb GB, Ellis JA. Knowledge taught in school: What is remembered? Rev Educ Res. 1994;64:25386.

12. Othman GQ, Ibrahim MIM, Alshakka M, Ansari M, Al-Qadasi F, Halboup AM. Knowledge and perception about pharmacovigilance among pharmacy students of universities in Sana'a Yemen. J Clin Diagn Res. 2017;11(6):FC09-13.

13. Loprinzi PD, Frith E. The Role of sex in memory function: considerations and recommendations in the context of exercise. J Clin Med. 2018;7(6).

14. Torwane NA, Hongal S, Gouraha A, Saxena E, Chavan K. Awareness related to reporting of adverse drug reactions among health caregivers: A crosssectional questionnaire survey $\mathrm{J}$ Nat Accred Board Hosp Healthcare Providers. 2015;2:23-9.

15. Bharadwaj V, Budania N, Mondal, Yadav V, Sharma P. A survey to assess the awareness of adverse drug reactions and pharmacovigilance practices among healthcare professionals in a tertiary care hospital. Int J Med Res Prof. 2016;2(1):134-7.

Cite this article as: Honnagangadevaru LS, Lakshmanegowda K. The short and long-term impact of educational intervention on pharmacovigilance awareness in second year MBBS students: a questionnaire-based survey. Int J Basic Clin Pharmacol 2020;9:590-4. 